\title{
Exploring the story structure of branching books
}

\author{
Diamantis Sellis \\ sellisd@gmail.com \\ CosmoTech Lyon, France
}

\begin{abstract}
Works of interactive fiction have been undergoing an expansive growth recently. However our understanding of the laws and patterns governing their design, structure and dynamics seem to be lagging behind. I here focus on the sub-genre of branching story books and develop tools to explore the structure of the story plot.
\end{abstract}

\section{Introduction}

Stories change and evolve through time as they are transmitted from person to person through generations. As a result, we observe a diversity of alternative stories with minor or large differences in both plot and motifs. A particular type of diversity is found in the genre of interactive fiction (broadly defined such as to include branching story books and games). A single work of interactive fiction has an inherent diversity of story plots as it contains multiple parallel alternative stories. These two types of diversity are not necessarily subject to the same laws and patterns of evolution although there might be some interesting parallels. The diversity of evolving stories has been the subject of attention in some recent studies such as Tehrani [2013] and Karsdorp and van den Bosch [2016]. The diversity of alternative plots in the genre of interactive fiction, to my knowledge, has been very scarcely explored.

A branching story book is a story book in which multiple alternative plots are written, potentially leading to multiple different endings. Such books are organized in a number of discrete story units, called storylets [Kreminski and Wardrip-Fruin, 2018], that combined form a complete plot. Often a storylet is a single page of the book, at the end of each page the reader can make a choice affecting the story plot. Depending on the choice the reader is directed to continue reading in a different storylet (different page) up until the end of the story plot. Starting over the book and making different choices can lead the reader to traverse different storylets and potentially reach a different end. Branching story books (also known as ergodic literature [Aarseth, 1997]) are a type of gamebook, a genre popularized during the '80s with the very influential series of "create your own adventure" books (for the history of the genre see Plowman [2015]). These where followed more recently by hyperlink stories and various games in which the reader/player makes choices affecting the plot of the story or the game (for a history of the interactive fiction works genre see Short [2016]). 
Inherent in a branching story book is the fact that a potentially very large number of alternative scenarios exist in a single story book. Therefore, understanding some aspects of the story structure could benefit by a data science network analysis. Various approaches of network analyses and data science in literature have been already implemented [Moretti, 2011, Underwood et al., 2018]. However, in the field of branching story books, to my knowledge, there are limited studies with few notable exceptions [Kostakis T, 2007, Kontopoulou et al., 2012, 2013].

To fill in this gap I develop tools to analyze and visualize different alternative paths and apply them to two branching books.

\section{Data and Methods}

To analyze the story structures I create a graphs representation, the story graph, on which I performed a number of network analysis steps.

\subsection{Data sources and coding}

I used as data sources a Greek and a French children's books with branching stories: "Ta ogdonta ochto ntolmadakia" by Evgenios Trivizas [Trivizas, 2006] and "Orient-express !" by Chedru Delphine [Delphine, 2014]. In Trivizas [2006] the storylets (story units) are single pages while in Delphine [2014] a storylet corresponds to a two-page spread (two facing pages). I constructed an edgelist for each book where source and target nodes are linked storylets. I coded a second dataset with storylet attributes. One attribute is the ending type, which indicates if the end corresponds to a happy ending or a sad ending of the story. In [Delphine, 2014] there is a single true happy ending of the story however there are also a number of pages corresponding to a bad ending where the goal of the quest is not achieved. These bad endings are not final and the reader is directed back to almost the beginning of the story having the ability to follow a different path. I coded such storylets as bad endings, even if they are not really the end of the story. Another attribute (only in [Trivizas, 2006]) is whether the storylet has a pause in the narrative flow. In Trivizas [2006] a number of pages do not have multiple questions leading to alternative pages with alternative plots. Instead, a pause is proposed with two different forms. In the one case a blank square is presented in which the reader can fill-in the story ending and if desired continue to another page where the story continues. In the other case the question to the user is "are you bored?" if the answer is "yes" an activity outside the book is proposed and if the answer is "no" a page link for the continuation of the story is proposed.

\subsection{Graph simplification}

To remove cycles in the story graph I perform a depth first search during which a stack of visited nodes is created. The stack is used to check if a node has been already visited in thus cycles are avoided. 


\subsection{Path clustering}

The number of all possible alternative story plots is potentially very large. Each of the alternative plots corresponds to a unique path in the story graph. Not all alternative paths represent a major plot difference. Differences in the content of different nodes (the plot of the story) could possible be also reflected in the structure of the graph. To identify such main branching paths or sub-stories I implemented a path clustering method in which all paths are compared using a similarity index. Consider a path $P_{i}$ as a set of nodes $\left(n_{1}, n_{2}, \ldots, n_{k}\right)$. I define the similarity index $S_{i j}$ of two paths $P i$ and $P j$ as:

$$
S_{i j}=\frac{|P i \cap P j|}{|P i \cup P j|}
$$

where $|P i|$ indicates the cardinality of a set. The similarity index is a measure of nodes that are shared between two paths. It is 1 for identical paths and 0 for completely different paths (paths without shared nodes).

I perform iteratively the path clustering by calculating the pairwise similarity index for all pairs of paths. Pairs with similarity under a user specified threshold are considered part of the same cluster and one out of the two is randomly chosen to represent the cluster in the following iteration. Iterations of the clustering continue until all remaining paths are more different than the chosen threshold for the similarity index.

\subsection{Tools for story structure exploration}

I developed tools for exploring the structure of branching stories or games implemented in python v.3.6.6 [Python Software Foundation] using the modules pandas v.0.19.2 [McKinney] and numpy v.1.11.3 [Oliphant, 2006]. Graph visualization was performed with Graphviz v.2.40.1 [Ellson et al., 2003]. Statistical analyses and plots were performed in R v. 3.5.0 [R Core Team, 2018] using the tidyverse packages v.1.2.1 [Wickham, 2017]. The source code is available at github (https://github.com/sellisd/storystructure) and as a package at pypi (https://pypi.org/project/storystructure/). Documentation and a jupyter notebook tutorial are available at (https://sellisd.github.io/storystructure/).

\section{Results and Discussion}

\subsection{Story structure visualization}

To visualize the structure of stories I plot a directed multigraph of each book. The nodes correspond to storylets and edges corresponds to links between them where the source is the storylet with the multiple option questions and the target is the storylet in which the plot continues (Figure 1). Sam [2017] has identified 8 basic patterns in works of interactive fiction that have different structural patterns and effects in the narrative. According to this tentative classification system Trivizas [2006] has a time-cave structure with multiple endings and an almost tree-like branching structure, while Delphine [2014] has quest structure a single ending with a small number of long branches. 


\subsection{Atypical structures and potential errors}

Some structural features of the graph can easily point towards errors inadvertently introduced during the writing of the story such as multiple starting points. In Trivizas [2006] one such node is easy to spot (page 102). It is impossible to reach to such hidden starting points of the story in a regular reading. Another feature of the structures that could possibly correspond to an error is the existence of multiple edges connecting two nodes. There are a number of such cases in Trivizas [2006] corresponding to two different choices that lead to the same page. Such redundant questions represent a deliberate choice of the author, for example in page 17 the choice is between the hero meeting a hungry bear or meeting a fasted bear and both answers lead to the same page (page 36) where the hero encounters a bear that is both hungry and fasted at the same time. A third type of potential errors are cycles leading to a never-ending story. There are cyclical paths in the graph ranging from self-loops to larger paths. The self-loops are due to the way in which the coding of the graph was made. They correspond to questions in which only the correct answer leads to a new page while a wrong answer forces the reader to stay in the same page. There is also a number of cycles of size 2, these are cycles that involve a "pause" page, a page in which the reader is asked to draw or write the continuation of the story and then to continue on the source page (e.g. pages 88 and 42). Finally, there are larger cycles involving more than two edges which are part of the story plot. For example in pages 94 and 147 the reader is directed from one page to another and vice versa as well as to page 108 . The reader must chose the answer leading to page 108 in order to break the cycle and let the story continue.

\subsection{Enumerating all possible paths}

To enumerate all possible stories (all possible traversals of the story tree) a number of graphs simplification steps are necessary. These include the removal of secondary roots, double edges and cycles. In Trivizas [2006] there are 1762 total possible paths, $64.6 \%$ of which have a happy ending. The average number of storylets per path is $21 \pm 5$ for stories with good endings and $18 \pm 4$ for stories with bad endings. In Delphine [2014] there are 23 total possible paths and the average number of storylets per path is $13 \pm 1$. The average number of storylets per path is close to the number of pages in most children's books (Figures 2 and 3, Table 1). Due to historical reasons children's picture books often follow the standard format of 32 pages. Ignoring the cover page, dedications etc, the actual text spans about $27-28$ pages or 14 spreads (double page illustrations) [Pattison, 2016]. It is also noteworthy that the length of paths with bad endings is less than the length of paths with good endings $\left(p<2.2 \cdot 10^{-16}\right.$, one-sided Welch Two Sample t-test), this would facilitate a reader to easily restart restart the book in order to reach a good ending.

The linear layout of a physical book necessitates the placement of pages in a linear order despite the fact that the book is going to be read in a nonlinear manner. Following the links in the story graph the reader has to turn pages ahead or backwards after making each choice. The number of pages the reader has to flip through is determined by the ordering of the pages. In Trivizas [2006] the number of pages is roughly normally distributed and can extend up to about 100 pages forward or backward. On the contrary in Delphine [2014] the reader 


\begin{tabular}{|c|c|c|c|}
\hline $\begin{array}{l}\text { Number of } \\
\text { Pauses }\end{array}$ & Story Ending & Good Ending & Bad Ending \\
\hline & 0 & 24 & 50 \\
\hline & 1 & 66 & 63 \\
\hline & 2 & 63 & 40 \\
\hline & 3 & 118 & 54 \\
\hline & 4 & 224 & 228 \\
\hline & 5 & 128 & 448 \\
\hline & 6 & - & 256 \\
\hline
\end{tabular}

Table 1: Number of alternative stories with none or one pause by type of story ending

mostly turns a single page at a time and only when approaching the end of a story path might have to flip multiple pages backwards (Figures 4).

\subsection{Major sub-plots}

Not all paths represent major alternative story versions. To identify the major sub-stories I used a path clustering method that removes very similar paths preserving the most diverse story paths. The process is iterative and the result depends on the threshold defined for the difference between two paths in order to merge them. The choice of the threshold to use is not straightforward and a priori there is not a single value that would guarantee the identification of major subplots. To identify if such a value exists, and if it is the same for both books, a series of cutoff values was explored. For each threshold I counted the number of alternative paths remaining and plot their relationship. As the threshold increases the number of alternative paths decreases. In Delphine [2014] the datapoints are much less than in Trivizas [2006] so the difference in the shape of the curve could either be due to sampling size or to a true underlying difference in the structure of the two stories. In Trivizas [2006] the number of alternative paths decreases with the increase of the cutoff size almost linearly in a double$\log$ scale. This indicates counter-intuitively that there is not a single scale of major subplots but a continuum of ever finer sub-plots. On the contrary in Delphine [2014] there is a sudden drop in the number of subplots between $10 \%$ and $20 \%$ and a second one between $40 \%$ and $50 \%$. The second drop leads to a degenerate structure with just a single path. Clustering the paths with a cutoff of $10 \%$ produces a simplified structure in both stories. However, based on the distributions this clustered structure truly captures the major sub-plots only in [Delphine, 2014] (Figures 5 and 6)

\subsection{Outlook}

In this analysis I applied some data analytic approaches to explore the structure of two branching books. Although important intuitions can come out of this approach it is far from a full analyses and completely ignores the content of the stories. As an approach it is therefore envisioned to be used as a complementary tool to rapidly gain intuition about the basic structure of branching stories. 


\section{Acknowledgements}

I would like to thank Natalia Chousou-Polydouri and Efstratios Gallopoulos for helpful comments and feedback on the manuscript.

\section{References}

Espen J. Aarseth. Cybertext: Perspectives on Ergodic Literature. Johns Hopkins University Press, 1997. ISBN 0801855799.

Chedru Delphine. Orient-express! Helium, 2014. ISBN 233002875X.

John Ellson, Emden R. Gansner, Eleftherios Koutsofios, Stephen C. North, and Gordon Woodhull. Graphviz and dynagraph - static and dynamic graph drawing tools. In Graph drawing software, pages 127-148. Springer-Verlag, 2003.

Folgert Karsdorp and Antal van den Bosch. The structure and evolution of story networks. Royal Society Open Science, 3(6):160071, jun 2016. doi: 10.1098/rsos. 160071 .

Eugenia-Maria Kontopoulou, Maria Predari, Thymios Kostakis, and Efstratios Gallopoulos. Graph and matrix metrics to analyze ergodic literature for children. In Proceedings of the 23rd ACM conference on Hypertext and social media - HT '12, New York, New York, USA, 2012. ACM Press. doi: 10.1145/2309996.2310018.

Eugenia-Maria Kontopoulou, Maria Predari, and Efstratios Gallopoulos. Onomatology and content analysis of ergodic literature. In 24th ACM conference on Hypertext and social media "Narrative and Hypertext" Workshop, 2013.

Gallopoulos E Kostakis T. "The 88 Ntolmadakia" eigenvector and beyond: Link Analysis and Linear Algebra on children's books, 05 2007. HPCLAB-SCG 07/04-07, presented at 1st Greek Panhellenic Student Conference "EYRHKA 2007" held with 11th Panhellenic Conference on Informatics (PCI 2007), Patras.

Max Kreminski and Noah Wardrip-Fruin. Sketching a map of the storylets design space. In Interactive Storytelling: Proceedings of the 11th International Conference on Interactive Digital Storyteling, ICIDS Dublin, Ireland, pages 160-164. Springer, 2018.

Wes McKinney. Data structures for statistical computing in python. In Proceedings of the 9th Python in Science Conference, pages 51-56.

Franco Moretti. Network theory, plot analysis, May 2011. URL https://litlab.stanford.edu/LiteraryLabPamphlet2.pdf.

Travis Oliphant. NumPy: A guide to NumPy. USA: Trelgol Publishing, 2006. URL http://www.numpy.org/. [Online; accessed 2019-01-15].

Darcy Pattison. How to write a children's picture book : tips $\mathbb{E}$ tricks for writing illustrated picture books for kids. Mims House, Little Rock, Arkansas, 2016. ISBN 0985213485. 
Martin Plowman. A brief history of gamebooks, 2015. URL A Brief History Of Gamebooks.

Python Software Foundation. Python language reference, version 3.0. http://www.python.org.

R Core Team. R: A Language and Environment for Statistical Computing. R Foundation for Statistical Computing, Vienna, Austria, 2018. URL https://www.R-project.org/.

Ashwell Kabo Sam. Standard patterns in choice-based games. https://heterogenoustasks . wordpress.com/2015/01/26/standard-patterns-in-choice-based-gan 2017.

Emily Short. Brief bibliography about if history, 2016. URL https://emshort.blog/2016/04/02/brief-bibliography-about-if-history.

Jamshid J. Tehrani. The Phylogeny of Little Red Riding Hood. PLoS ONE, 8 (11):e78871, nov 2013. doi: 10.1371/journal.pone.0078871.

Eugenios Trivizas. Ta ogdonta ochto ntolmadakia. Kalentis, 2006. ISBN 9602190809

Ted Underwood, David Bamman, and Sabrina Lee. The Transformation of Gender in English-Language Fiction. Journal of Cultural Analytics, 02 2018. doi: 10.22148/16.019. URL http://culturalanalytics.org/2018/02/the-transformation-of-gender-in-english-language-fi

Hadley Wickham. tidyverse: Easily Install and Load the 'Tidyverse', 2017. URL https://CRAN.R-project.org/package=tidyverse. $\mathrm{R}$ package version 1.2.1. 
Figures

A

B
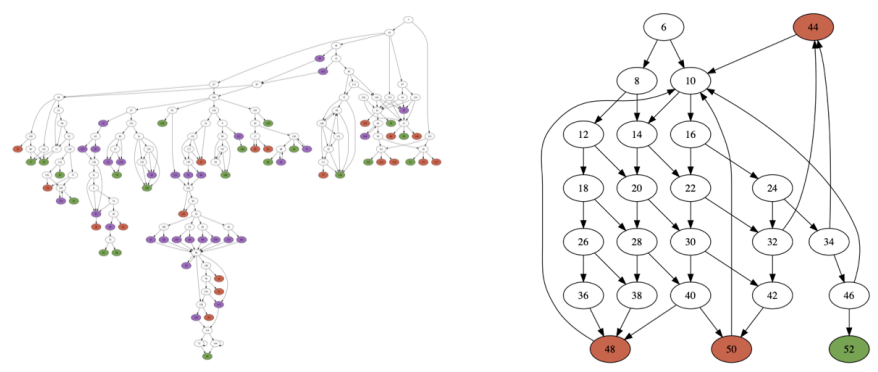

Figure 1: Structure of story graph for the two books analyzed. Node numbers correspond to pages. The color of nodes represent their attributes, green and red for happy and sad endings correspondingly, and violet for pause pages. A: Trivizas [2006], B: Delphine [2014] 

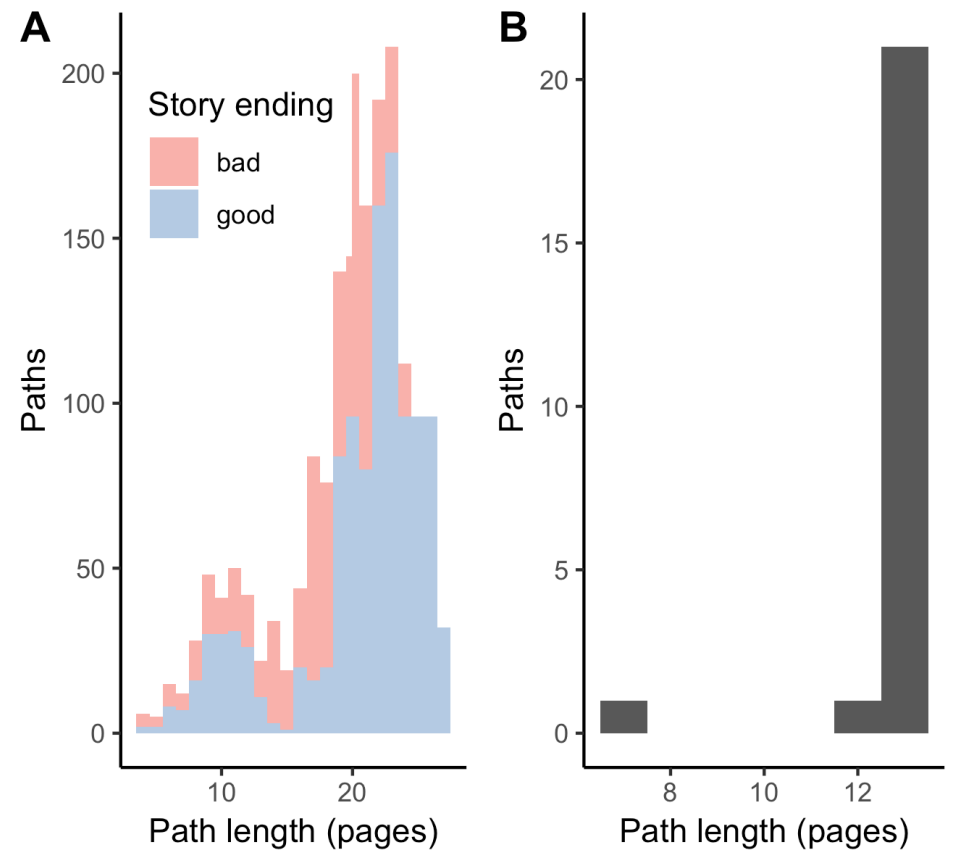

Figure 2: Distribution of path lengths (number of pages). A: Trivizas [2006], B: Delphine [2014]

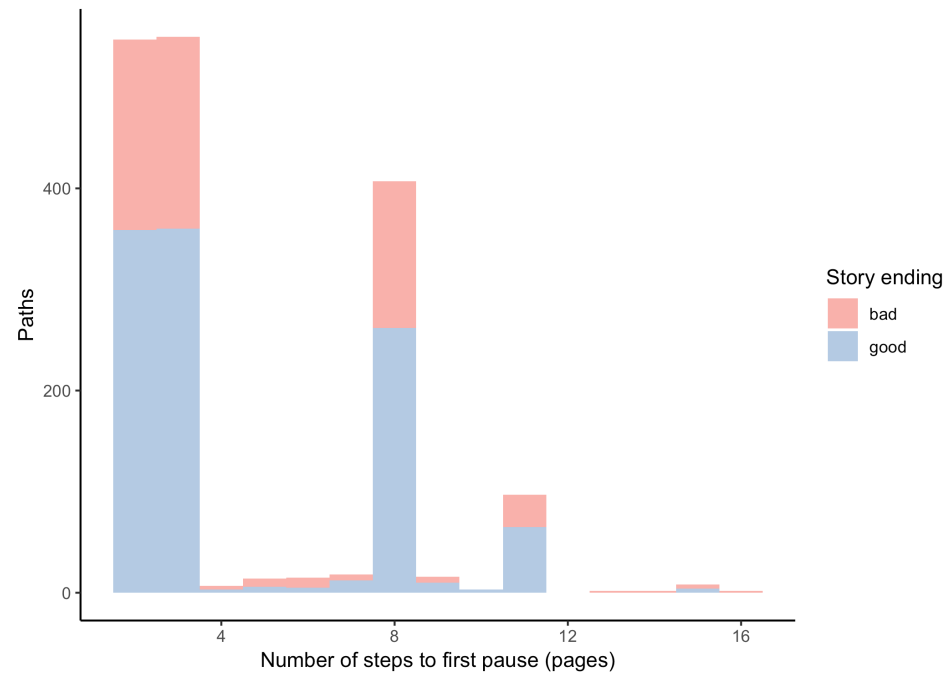

Figure 3: Distribution of story length (number of pages) from beginning to first pause grouped by the type of story ending 

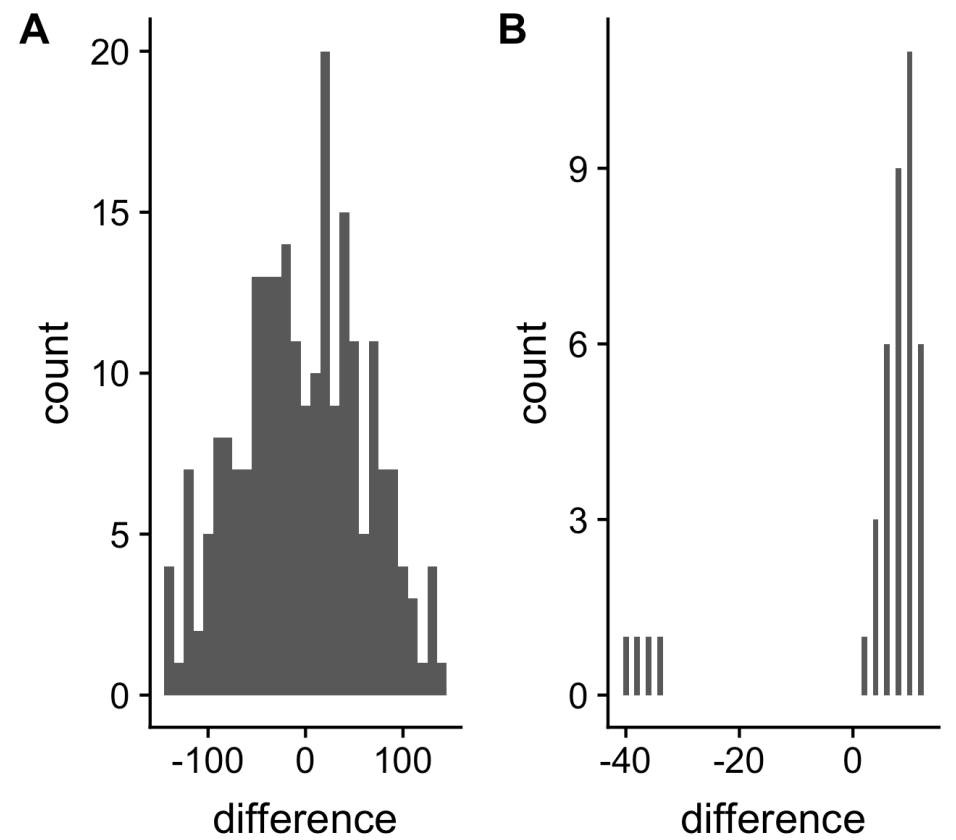

Figure 4: Distribution of the number of pages separating two consecutive nodes in a story path, negative values represent turning pages backwards. Note that the axes scales in the two panels are different.

A

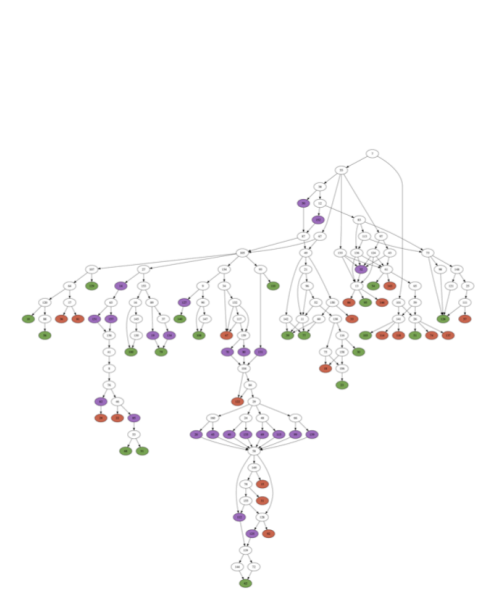

B

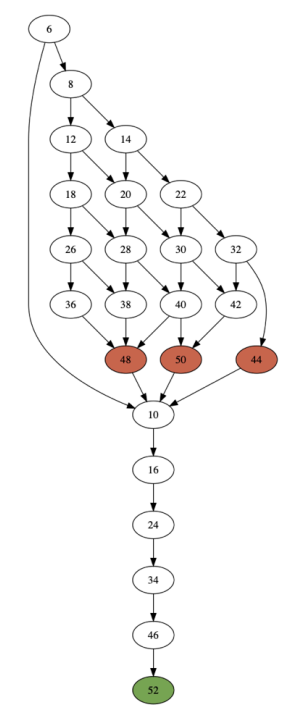

Figure 5: Graph structure with a threshold at 10\% A:Trivizas [2006], B:Delphine [2014] 

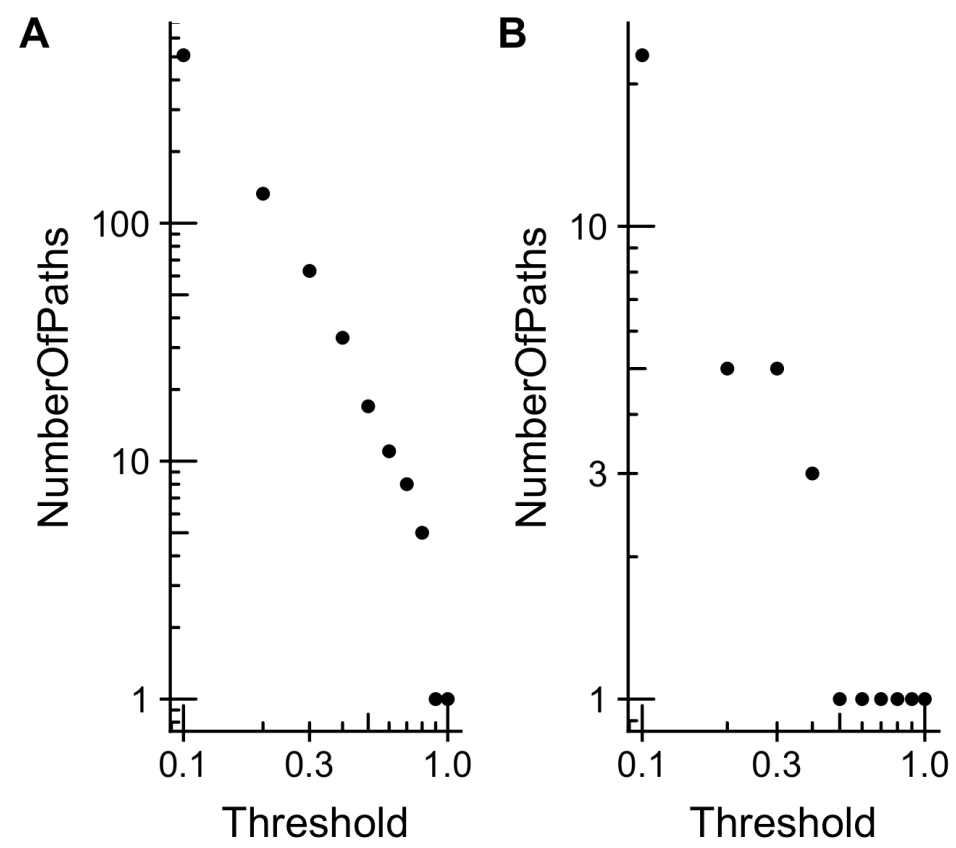

Figure 6: Effect of the threshold value on the number of major subplots. A:Trivizas [2006], B:Delphine [2014] 